\title{
Nonlinear Integro-differential Equations by Differential Transform Method with Adomian Polynomials
}

\author{
S. H. Behiry \\ General Required Courses Department, Jeddah Community College, King Abdulaziz University, Jeddah 21589, KSA \\ Email Address: salah_behiry@hotmail.com
}

Received: 25 May. 2013, Revised: 20 Jun. 2013; Accepted: 13 Jul 2013

Published online: 1 Sep. 2013

\begin{abstract}
A modification of differential transformation method is applied to nonlinear integro-differential equations. In this technique, the nonlinear term is replaced by its Adomian polynomials for the index $\mathrm{k}$, and hence the dependent variable components are replaced in the recurrence relation by their corresponding differential transform components of the same index. Thus the nonlinear integro-differential equation can be easily solved with less computational work for any analytic nonlinearity due to the properties and available algorithms of the Adomian polynomials. Numerical simulations of integro-differential equations with different types of nonlinearity are treated and the proposed technique has provided good results.
\end{abstract}

Keywords: Differential transform method; nonlinear integro-differential equations; Adomian polynomials.

\section{Introduction}

Integral and integro-differential equations play an important role in characterizing many social, biological, physical and engineering problems; for more details see [1-3] and references cited therein. Nonlinear integral and integro-differential equations are usually hard to solve analytically and exact solutions are rather difficult to be obtained. In literature nonlinear integral and integro-differential equations can be solved by many numerical methods such as the Legendre wavelets method [4], the Haar functions method [5, 6], the linearization method [7], the finite difference method [8], the Tau method [9, 10], the hybrid Legendre polynomials and block-pulse functions [11], the Adomian decomposition method $[12,13]$, the Taylor polynomial method [14-16] and the differential transform method [17].

The differential transform method (DTM) has been proved to be efficient for handling nonlinear problems, but the nonlinear functions used in these studies are restricted to polynomials and products with derivatives [17-21]. For other types of nonlinearities, the usual way to calculate their transformed functions as introduced by [22] is to expand the nonlinear function in an infinite power series then take the differential transform of this series. The problem with this approach is that the massive computational difficulties will arise in determining the differential transform of nonlinear function while working with this infinite series. Another approach for obtaining the differential transform of nonlinear terms is the algorithm in [23]. It is based on using the properties of differential transform and calculus to develop a canonical equation. Then this equation is solved for the required differential transform of nonlinear term. But, as seen in the simple examples in section 3 in [23], the algorithm requires a sequence of differentiation, algebraic manipulations and computations of differential transform for other functions which is more difficult for the case of composite nonlinearities.

In this work, we introduce a comprehensive and more efficient approach for using the DTM to solve nonlinear integro-differential equations; the idea is based on the methodology in [24]. The nonlinear function is replaced by its Adomian polynomials and then the dependent variable components are replaced by their corresponding differential transform component of the same index. This technique benefited the 
properties of the Adomian polynomials and the efficient algorithm to generate them quickly as in the work [25-27].

\section{Differential Transform Method}

The basic definition and the fundamental theorems of the differential transformation and its applicability for various kinds of differential and integral equations are given in [18-22]. A review of differential transformation is presented here. The transformation of the $\mathrm{k}$-th derivative of a function in one variable is as follows

$$
Y(k)=\frac{1}{k !}\left[\frac{d^{k}}{d x^{k}} y(x)\right]_{x=x_{0}},
$$

and the inverse transformation is defined by

$$
y(x)=\sum_{k=0}^{\infty} Y(k)\left(x-x_{0}\right)^{k} .
$$

In this work, we use lower case letter for the original functions and upper case letter stand for the transformed functions. The following theorems can be deduced from equations (1) and (2).

Theorem 1. If $y(x)=f(x) \pm h(x)$, then $Y(k)=F(k) \pm H(k)$.

Theorem 2. If $y(x)=c f(x)$, then $Y(k)=c F(k)$, where $c$ is a constant.

Theorem 3. If $y(x)=f^{(n)}(x)$, then $Y(k)=\frac{(k+n) !}{k !} F(k)$.

Theorem 4. If $y(x)=f(x) h(x)$, then $Y(k)=\sum_{k_{1}=0}^{k} F\left(k_{1}\right) H\left(k-k_{1}\right)$.

Theorem 5. If $y(x)=x^{m}$, then $Y(k)=\delta(k-m)$, where $\delta(k-m)=\left\{\begin{array}{ll}1, & k=m \\ 0, & k \neq m\end{array}\right.$.

Theorem 6. If $y(x)=\int_{x_{0}}^{x} f(t) d t \quad$, then $Y(k)=\frac{F(k-1)}{k}, \quad k \geq 1$.

Theorem 7. If $y(x)=\int_{x_{0}}^{x} f_{1}(t) f_{2}(t) d t \quad Y(k)=\frac{1}{k} \sum_{k_{1}=0}^{k-1} F_{1}\left(k_{1}\right) F_{2}\left(k-k_{1}-1\right), \quad k \geq 1$.

Theorem 8. If $y(x)=f(x) \int_{x_{0}}^{x} h_{1}(t) h_{2}(t) d t$, then

$$
\begin{gathered}
y(x)=f(x) \int_{x_{0}}^{x} h_{1}(t) h_{2}(t) d t, \text { then } \\
\sum_{k_{2}=1}^{k} \sum_{k_{1}=1}^{k_{2}} \frac{1}{k_{2}} H_{1}\left(k_{1}-1\right) H_{2}\left(k_{2}-k_{1}\right) F\left(k-k_{2}\right), \quad k \geq 1
\end{gathered}
$$

The following relation is quite useful in the solution of Fredholm integrals; it can be obtained from Theorem7 and equation (2), i. e. 


$$
\int_{a}^{b} f_{1}(t) f_{2}(t) d t=\sum_{k_{1}=1}^{\infty}\left\{\frac{1}{k}\left[\left(b-x_{0}\right)^{k}-\left(a-x_{0}\right)^{k}\right] \sum_{k_{1}=0}^{k-1} F_{1}\left(k_{1}\right) F_{2}\left(k-k_{1}-1\right)\right\} .
$$

\section{The Modified Differential Transform Method}

In this section, we introduced a reliable and efficient algorithm to calculate the differential transform of a nonlinear function $g(y)$. This nonlinear function can be decomposed as

$$
g(y)=\sum_{n=0}^{\infty} A_{n},
$$

where $A_{n}, n \geq 0$ are the Adomian polynomials determined formally as follows [12, 28]

$$
A_{n}=\frac{1}{n !}\left[\frac{d^{n}}{d \lambda^{n}}\left[g\left(\sum_{i=0}^{\infty} \lambda_{i} y_{i}\right)\right]\right]_{\lambda=0} .
$$

The Adomian polynomials of $g(y)$ are introduced as

$$
\begin{aligned}
& A_{0}=g\left(y_{0}\right), \\
& A_{1}=y_{1} g^{(1)}\left(y_{0}\right), \\
& A_{2}=y_{2} g^{(1)}\left(y_{0}\right)+\frac{1}{2 !} y_{1}^{2} g^{(2)}\left(y_{0}\right), \\
& A_{3}=y_{3} g^{(1)}\left(y_{0}\right)+y_{1} y_{2} g^{(2)}\left(y_{0}\right)+\frac{1}{3 !} y_{1}^{3} g^{(3)}\left(y_{0}\right), \\
& A_{4}=y_{4} g^{(1)}\left(y_{0}\right)+\left(y_{1} y_{3}+\frac{1}{2 !} y_{2}^{2}\right) g^{(2)}\left(y_{0}\right)+\frac{1}{2 !} y_{1}^{2} y_{2} g^{(3)}\left(y_{0}\right)+\frac{1}{4 !} y_{1}^{4} g^{(4)}\left(y_{0}\right) \\
& A_{5}=y_{5} g^{(1)}\left(y_{0}\right)+\left(y_{2} y_{3}+y_{1} y_{4}\right) g^{(2)}\left(y_{0}\right)+\frac{1}{2 !}\left(y_{1}^{2} y_{3}+y_{1} y_{2}^{2}\right) g^{(3)}\left(y_{0}\right) \\
& \quad+\frac{1}{3 !} y_{1}^{3} y_{2} g^{(4)}\left(y_{0}\right)+\frac{1}{5 !} y_{1}^{5} g^{(5)}\left(y_{0}\right), \text { and so on. }
\end{aligned}
$$

Hence the differential transform components of $g(y)$ are computed by utilizing their properties, they can be written in the following form (for $x=0$ )

$$
\begin{aligned}
& G(0)=g(Y(0)) \\
& G(1)=Y(1) g^{(1)}(Y(0)), \\
& G(2)=Y(2) g^{(1)}(Y(0))+\frac{1}{2 !} Y^{2}(1) g^{(2)}(Y(0)) \\
& G(3)=Y(3) g^{(1)}(Y(0))+Y(1) Y(2) g^{(2)}(Y(0))+\frac{1}{3 !} Y^{3}(1) g^{(3)}(Y(0)),
\end{aligned}
$$




$$
\begin{aligned}
& G(4)=Y(4) g^{(1)}(Y(0))+\left(Y(1) Y(3)+\frac{1}{2 !} Y^{2}(2)\right) g^{(2)}(Y(0)) \\
&+ \frac{1}{2 !} Y^{2}(1) Y(2) g^{(3)}(Y(0))+\frac{1}{4 !} Y^{4}(1) g^{(4)}(Y(0)) \\
& G(5)=Y(5) g^{(1)}(Y(0))+(Y(2) Y(3)+Y(1) Y(4)) g^{(2)}(Y(0)) \\
&+\frac{1}{2 !}\left(Y^{2}(1) Y(3)+Y(1) Y^{2}(2)\right) g^{(3)}(Y(0))+\frac{1}{3 !} Y^{3}(1) Y(2) g^{(4)}(Y(0)) \\
&+\frac{1}{5 !} Y^{5}(1) g^{(5)}(Y(0)), \text { and so on. }
\end{aligned}
$$

The advantage of using this algorithm compared to the algorithm suggested in [23] for computing differential transformation of nonlinear functions, is this algorithm deals directly with nonlinear function of the problem in hand in its form without any differentiation, algebraic manipulation and no need to compute the differential transform of other functions to obtain the required one.

\section{Applications and Numerical Results}

In this section, we implement the proposed method on some different examples with different types of nonlinearity. All algebraic computations are executed using MATHEMATICA software package.

Example 1. Consider the nonlinear Volterra integro-differential equation

$$
y^{\prime \prime}(x)+y^{\prime}(x) y(x)+y(x)=\cos 2 x+x^{3}-x^{2} \int_{0}^{x} \frac{1+\sin 2 t}{y^{2}(t)} d t \quad, 0 \leq x \leq 1,
$$

with the initial conditions

$$
y(0)=1 \text { and } y^{\prime}(0)=1 .
$$

The differential transformation of equation (8) and the initial conditions (9) are

$$
\begin{aligned}
Y(k+2)=\frac{k !}{(k+2) !}\left[\frac{2^{k}}{k !} \cos (\pi k / 2)\right. & +\delta(k-3)-\sum_{m=0}^{k}(m+1) Y(m+1) Y(k-m)-Y(k) \\
& \left.-\frac{G(k-3)}{k-2}-\frac{1}{k-2} \sum_{m=1}^{k-2} \frac{2^{m-1}}{(m-1) !} \sin \left(\frac{\pi(m-1)}{2}\right) G(k-m-2)\right],
\end{aligned}
$$

where $G(k)$ are the differential transform (Adomian polynomials) of the nonlinear function $g(y)=y^{-2}$, and

$$
Y(0)=1 \text { and } Y(1)=1 \text {. }
$$

Using the relations in (7) the Adomian polynomials for this nonlinear function are

$$
\begin{aligned}
& G(0)=g(Y(0))=1, \\
& G(1)=-2 Y(1),
\end{aligned}
$$




$$
\begin{aligned}
& G(2)=-2 Y(2)+3 Y^{2}(1) \\
& G(3)=-2 Y(3)+6 Y(1) Y(2)-4 Y^{3}(1) \text { and } \\
& G(4)=-2 Y(4)-2 Y(1) Y(3)-Y^{2}(2)+3 Y^{2}(1) Y(2)+5 Y^{4}(1) .
\end{aligned}
$$

Utilizing the recurrence relation (10), the transformed initial conditions (11) and the Adomian polynomials (12), $Y(k)$ are evaluated. Hence using the inverse transformation in equation (2), the following series solution up to $O\left(x^{10}\right)$ can be obtained

$$
y(x)=1+x-\frac{x^{2}}{2 !}-\frac{x^{3}}{3 !}+\frac{x^{4}}{4 !}+\frac{x^{5}}{5 !}-\frac{x^{6}}{6 !}-\frac{x^{7}}{7 !}+\frac{x^{8}}{8 !}+\frac{x^{9}}{9 !}+O\left(x^{10}\right)
$$

For sufficiently large number of terms, the closed form of the solution is $y(x)=\sin x+\cos x$, which is the exact solution. Table 1 shows the absolute relative error (Abs. rel. err.) obtained for three various numbers of terms and at some test points.

Table 1: Numerical comparison of results in Example 1.

\begin{tabular}{|l|l|l|l|}
\hline$x$ & Abs. rel. err., (5 Terms) & Abs. rel. err., (10 Terms) & Abs. rel. err., (15 Terms) \\
\hline 0.2 & $7.75099 \mathrm{E}-08$ & 0 & 0 \\
\hline 0.4 & $4.57623 \mathrm{E}-06$ & $7.74161 \mathrm{E}-13$ & 0 \\
\hline 0.6 & $5.02971 \mathrm{E}-05$ & $6.19746 \mathrm{E}-11$ & 0 \\
\hline 0.8 & $2.83721 \mathrm{E}-04$ & $1.41455 \mathrm{E}-09$ & $1.25621 \mathrm{E}-15$ \\
\hline
\end{tabular}

Example 2. Consider the nonlinear Volterra integro-differential equation

$$
y^{\prime \prime}(x)-6 y(x)=-4+8 \int_{0}^{x} t y(t) \ln y(t) d t \quad 0 \leq x \leq 1,
$$

with the initial conditions

$$
y(0)=1 \text { and } y^{\prime}(0)=0 .
$$

Application of the differential transform to equation (13) gives

$$
(k+1)(k+2) Y(k+2)-6 Y(k)=-4 \delta(k)+\frac{8}{k} G(k-2),
$$

where $G(k)$ are the Adomian polynomials of the nonlinear function $g(y)=y \ln y$.

Substitute $k=0$ and $k=1$ into equation (15), one can get the following relations

$$
\begin{aligned}
& 2 Y(2)-6 Y(0)=-4, \\
& 6 Y(3)-6 Y(1)=0 .
\end{aligned}
$$

Also, for $k \geq 2$, equation (15) can be written as 


$$
Y(k+2)=\frac{1}{(k+1)(k+2)}\left[6 Y(k)+\frac{8}{k} G(k-2)\right] .
$$

The initial conditions in (14) are transformed by using (1) to

$$
Y(0)=1 \text { and } Y(1)=0 \text {. }
$$

Using the relations in (7), the Adomian polynomials for the nonlinear function of this example are

$$
\begin{aligned}
& G(0)=g(Y(0))=1 \\
& G(1)=Y(1) \\
& G(2)=Y(2)-(1 / 2) Y^{2}(1) \\
& G(3)=Y(3)-Y(1) Y(2)+(1 / 3) Y^{3}(1) \\
& G(4)=Y(4)-Y(1) Y(3)-(1 / 2) Y^{2}(2)+Y^{2}(1) Y(2)-(1 / 4) Y^{4}(1) \\
& G(5)=Y(5)-Y(2) Y(3)-Y(1) Y(4)+Y^{2}(1) Y(3)+Y(1) Y^{2}(2)-Y^{3}(1) Y(2)+(1 / 5) Y^{5}(1) \text { and } \\
& G(6)=Y(6)-(1 / 2) Y^{2}(3)-Y(2) Y(4)-Y(1) Y(5)+(1 / 2) Y^{2}(1) Y^{2}(2)+(1 / 3) Y^{3}(1) Y(3) \\
& \quad-(3 / 2) Y^{2}(1) Y^{2}(2)-Y^{3}(1) Y(3)+Y^{4}(1) Y(2)-(1 / 6) Y^{6}(1)
\end{aligned}
$$

Utilizing the above relations (16)-(20), one can easily solve for ${ }^{Y(k)}$. Using the inverse transformation rule in equation (2), the following series solution for equation (13) up to $O\left(x^{11}\right)$ is

$$
y(x)=1+x^{2}+\frac{1}{2} x^{4}+\frac{1}{6} x^{6}+\frac{1}{24} x^{8}+\frac{1}{120} x^{10}+O\left(x^{11}\right) .
$$

For sufficiently large number of terms, the closed form of the solution is $y(x)=e^{x^{2}}$, which is the exact solution. Table 2 shows the absolute relative error (Abs. rel. err.) obtained for three various numbers of terms and at some test points.

Table 2: Numerical comparison of results in Example 2.

\begin{tabular}{|l|l|l|l|}
\hline$x$ & Abs. rel. err., (5 Terms) & Abs. rel. err.,(10 Terms) & Abs. rel. err., (15 Terms) \\
\hline 0.2 & $8.25371 \mathrm{E}-10$ & 0 & 0 \\
\hline 0.4 & $7.64934 \mathrm{E}-07$ & $2.83821 \mathrm{E}-15$ & 0 \\
\hline 0.6 & $3.73777 \mathrm{E}-05$ & $7.26632 \mathrm{E}-12$ & 0 \\
\hline 0.8 & 5.27137 E-04 & $1.77822 \mathrm{E}-09$ & 0 \\
\hline
\end{tabular}

Example 3. Consider the nonlinear Volterra integro-differential equation

$$
6\left(x^{2}+1\right) y^{\prime}(x)=\left(x^{3}+3 x^{2}+6 x+6\right) e^{-x}+\int_{0}^{x} t^{3} e^{-\tan y(t)} d t \quad, \quad 0 \leq x \leq 1,
$$

with the initial condition 


$$
y(0)=0
$$

The differential transformation of equation (21) and the initial condition (22) are

$$
Y(k+1)=-\frac{k-1}{k+1} Y(k-1)+\frac{1}{6(k+1)}\left[\frac{(-1)^{k}\left(6-11 k+6 k^{2}-k^{3}\right)}{k !}+\frac{G(k-4)}{k}\right],
$$

where $G(k)$ are the Adomian polynomials of the nonlinear function $g(y)=e^{-\tan y}$, and

$$
Y(0)=0 \text {. }
$$

If we substitute $x=0$ into equation (21), we can get $y^{\prime}(0)=1$ and hence

$$
Y(1)=1 \text {. }
$$

The following system for $k=1,2,3, \cdots, 8$ is obtained from (23)

$$
\begin{aligned}
& Y(2)=0, \\
& Y(3)=-\frac{1}{3} Y(1), \\
& Y(4)=-\frac{2}{4} Y(2), \\
& Y(5)=-\frac{3}{5} Y(3)+\frac{1}{6(5)}\left[-\frac{6}{4 !}+\frac{G(0)}{4}\right], \\
& Y(6)=-\frac{4}{6} Y(4)+\frac{1}{6(6)}\left[\frac{24}{5 !}+\frac{G(1)}{5}\right], \\
& Y(7)=-\frac{5}{7} Y(5)+\frac{1}{6(7)}\left[-\frac{60}{6 !}+\frac{G(2)}{6}\right], \\
& Y(8)=-\frac{6}{8} Y(6)+\frac{1}{6(8)}\left[\frac{120}{7 !}+\frac{G(3)}{7}\right] \text { and } \\
& Y(9)=-\frac{7}{9} Y(7)+\frac{1}{6(9)}\left[-\frac{210}{8 !}+\frac{G(4)}{8}\right] .
\end{aligned}
$$

$G(k)$ can be obtained by using the relations in (7) for the function $g(y)=e^{-\tan y}$ as

$$
\begin{aligned}
& G(0)=e^{-\tan (Y(0))}=1, \\
& G(1)=Y(1) \\
& G(2)=Y^{2}(1)-Y(2), \\
& G(3)=-(1 / 2) Y^{3}(1)+Y(1) Y(2)-Y(3) \text { and }
\end{aligned}
$$




$$
G(4)=Y(1) Y(3)+(1 / 2) Y^{2}(2)-(3 / 2) Y^{2}(1) Y(2)+(3 / 8) Y^{4}(1)-Y(4)
$$

On solving the above systems for $Y(k)$, the series solution of equation (21) up to $O\left(x^{10}\right)$ is given by

$$
y(x)=x-\frac{x^{3}}{3}+\frac{x^{5}}{5}-\frac{x^{7}}{7}+\frac{x^{9}}{9}+O\left(x^{10}\right) .
$$

For sufficiently large number of terms, the closed form of the solution is $y(x)=\tan ^{-1} x$, which is the exact solution. Table 3 shows the absolute relative error (Abs. rel. err.) obtained for three various numbers of terms and at some test points.

Table 3: Numerical comparison of results in Example 3.

\begin{tabular}{|l|l|l|l|}
\hline$x$ & Abs. rel. err., (5 Terms) & Abs. rel. err.,(10 Terms) & Abs. rel. err., (15 Terms) \\
\hline 0.2 & $9.12337 \mathrm{E}-09$ & 0 & 0 \\
\hline 0.4 & $8.82908 \mathrm{E}-06$ & $4.80303 \mathrm{E}-10$ & 0 \\
\hline 0.6 & $4.68447 \mathrm{E}-04$ & $1.45549 \mathrm{E}-06$ & $5.92003 \mathrm{E}-10$ \\
\hline 0.8 & $7.53201 \mathrm{E}-03$ & $4.11299 \mathrm{E}-04$ & $2.95854 \mathrm{E}-07$ \\
\hline
\end{tabular}

Example 4. Let us consider the nonlinear Volterra integro-differential equation

$$
y^{\prime \prime}(x)-2 y(x) y^{\prime}(x)=-x+\int_{0}^{x} \frac{y^{\prime}(t)}{1+y^{2}(t)} d t, \quad 0 \leq x \leq 1,
$$

with the initial conditions

$$
y(0)=0 \text {, and } y^{\prime}(0)=1
$$

The differential transformation of equation (27) and the initial condition (28) are

$$
Y(k+2)=\frac{k !}{(k+2) !}\left[2 \sum_{m=0}^{k}(m+1) Y(m+1) Y(k-m)-\delta(k-1)+\frac{1}{k} \sum_{m=0}^{k-1}(m+1) Y(m+1) G(k-m-1)\right],
$$

where ${ }^{G(k)}$ are the Adomian polynomials of the nonlinear function $g(y)=\frac{1}{1+y^{2}}$, and

$$
Y(0)=0 \text { and } Y(1)=1 .
$$

$G(k)$ can be obtained by using the relations in (7) as

$$
\begin{aligned}
& G(0)=\left(1+Y^{2}(0)\right)^{-1}=1, \\
& G(1)=0, \\
& G(2)=-Y^{2}(1), \\
& G(3)=-2 Y(1) Y(2) \text { and }
\end{aligned}
$$


$G(4)=-2\left(Y(1) Y(3)+Y^{2}(2)\right)+Y^{4}(1)$

On solving the above systems (29)-(31) for ${ }^{(k)}$, the series solution of equation (27) up to $O\left(x^{10}\right)$ is given by

$$
y(x)=x+\frac{1}{3} x^{3}+\frac{2}{15} x^{5}+\frac{17}{315} x^{7}+\frac{62}{2835} x^{9}+O\left(x^{10}\right)
$$

For sufficiently large number of terms, the closed form of the solution is $y(x)=\tan x$, which is the exact solution. Table 4 shows the absolute relative error (Abs. rel. err.) obtained for three various numbers of terms and at some test points.

Table 4; Numerical comparison of results in Example 4.

\begin{tabular}{|l|l|l|l|}
\hline$x$ & Abs. rel. err., (5 Terms) & Abs. rel. err.,(10 Terms) & Abs. rel. err., (15 Terms) \\
\hline 0.2 & $9.10218 \mathrm{E}-10$ & 0 & 0 \\
\hline 0.4 & $9.40244 \mathrm{E}-07$ & $7.01122 \mathrm{E}-14$ & $6.49916 \mathrm{E}-15$ \\
\hline 0.6 & $5.50308 \mathrm{E}-05$ & $5.30862 \mathrm{E}-10$ & $4.48908 \mathrm{E}-12$ \\
\hline 0.8 & $9.98396 \mathrm{E}-04$ & $3.04049 \mathrm{E}-07$ & $2.23001 \mathrm{E}-10$ \\
\hline
\end{tabular}

Example 5. Let us consider the nonlinear Fredholm integro-differential equation [23]

$$
\left(x^{3}+1\right) y^{(4)}(x)-\frac{8}{315} y^{\prime}(x)=\frac{x^{2}}{8}+\int_{0}^{1} x t(x+t) y^{3}(t) d t, \quad 0 \leq x \leq 1
$$

with the initial conditions

$$
y(0)=-1, y^{\prime}(0)=0, y^{\prime \prime}(0)=2 \text { and } y^{\prime \prime \prime}(0)=0 .
$$

The differential transformation of equation (32) and its initial conditions (33) are

$$
Y(k+4)=\frac{k !}{(k+4) !}\left[-\frac{k !}{(k-3) !} Y(k)+\frac{8(k+1)}{315} Y(k+1)+\left(\frac{1}{8}+\alpha\right) \delta(k-2)+\beta \delta(k-1)\right]
$$

where

$$
\alpha=\int_{0}^{1} t y^{3}(t) d t \quad \beta=\int_{0}^{1} t^{2} y^{3}(t) d t
$$

and

$$
Y(0)=-1, Y(1)=0, Y(2)=1 \text { and } Y(3)=0 .
$$

To obtain $^{Y(4)}$, substitute $x=0$ into equation (32) and utilizing the transformation (1), hence

$$
Y(4)=0 \text {. }
$$

Substitute $k=1$ and $k=2$ into equation (34), one can get the following relations

$$
Y(5)=\frac{2}{4725}+\frac{\beta}{120} \text { and }
$$




$$
Y(6)=\frac{1}{2880}+\frac{\alpha}{360}
$$

The following recurrence relation can be obtained from equation (34)

$$
Y(k+4)=\frac{k !}{(k+4) !}\left[-\frac{k !}{(k-3) !} Y(k)+\frac{8(k+1)}{315} Y(k+1)\right], k \geq 3 \text {. }
$$

Utilizing relation (3) for (35), it can be shown that the following equalities hold for $\alpha$ and $\beta$

$$
\alpha=\sum_{k=2}^{N} \frac{G(k-2)}{k}, \quad \text {, and } \quad \sum_{k=3}^{N} \frac{G(k-3)}{k},
$$

where $N$ is a suitably large integer that represents the number of terms to be chosen, and $G(k)$ are the Adomian polynomials of the nonlinear function $g(y)=y^{3}$ as follow

$$
\begin{aligned}
& G(0)=Y^{3}(0)=-1, \\
& G(1)=-3 Y(1) \\
& G(2)=3 Y(2)+Y(1) Y(2)-3 Y^{2}(1), \\
& G(3)=3 Y(3)-6 Y(1) Y(2))+Y^{3}(1) \\
& G(4)=3 Y(4)-6 Y(1) Y(3)-3 Y^{2}(2)+3 Y^{2}(1) Y(2), \\
& G(5)=3 Y(5)-6(Y(2) Y(3)+Y(1) Y(4))+3\left(Y^{2}(1) Y(3)+Y(1) Y^{2}(2)\right) \text { and } \\
& G(6)=3 Y(6)-\left(3 Y^{2}(3)+6 Y(2) Y(4)+6 Y(1) Y(5)\right)+Y^{3}(2)+6 Y(1) Y(2) Y(3)+3 Y^{2}(1) Y(4) .
\end{aligned}
$$

Taking into account relations in (41), one can solve equations in (40) by taking $N=8$ to obtain the following results

$$
\alpha=-1 / 8 \text {, and } \beta=-16 / 315 \text {. }
$$

Substituting these values of $\alpha_{\text {and }}^{\beta}$ into equations (38) and (39), hence solving for $Y(k)$ the expansion for the unknown function $y(x)$ can be obtained, that is

$$
y(x)=-1+x^{2},
$$

which is the exact solution.

Example 6. Lastly, the following nonlinear system of Volterra integro-differential equations is considered [19, 29]

$$
\begin{aligned}
& u^{\prime \prime}(x)=1-\frac{1}{3} x^{3}-\frac{1}{2} v^{\prime 2}(x)+\frac{1}{2} \int_{0}^{x}\left[u^{2}(t)+v^{2}(t)\right] d t, \\
& v^{\prime \prime}(x)=-1+x^{2}-x u(x)+\frac{1}{4} \int_{0}^{x}\left[u^{2}(t)-v^{2}(t)\right] d t,
\end{aligned}
$$


with the initial conditions

$$
\begin{aligned}
& u(0)=1, \\
& u^{\prime}(0)=2, \\
& v(0)=-1, \\
& v^{\prime}(0)=0 .
\end{aligned}
$$

The differential transformation of the system (42) is given by

$$
\begin{gathered}
U(k+2)=\frac{k !}{(k+2) !}\left(\delta(k)-\frac{\delta(k-3)}{3}-\frac{G 1(k)}{2}+\frac{G 2(k-1)+G 3(k-1)}{2 k}\right), \\
V(k+2)=\frac{k !}{(k+2) !}\left(-\delta(k)-\delta(k-2)-U(k-1)+\frac{G 2(k-1)-G 3(k-1)}{4 k}\right),
\end{gathered}
$$

where $G 1(k), G 2(k)$ and $G 3(k)$ are the differential transforms of the nonlinear functions $g_{1}\left(v^{\prime}\right)=v^{\prime 2}$, $g_{2}(u)=u^{2}$ and $g_{3}(v)=v^{2}$, respectively. The initial conditions in equations (43) are transformed using equation (1) as follows

$$
U(0)=1, \quad U(1)=2, \quad V(0)=-1 \text { and } V(1)=0 .
$$

Utilizing the relations in (7), we obtained

$$
\begin{aligned}
& G 1(0)=V^{2}(1), \\
& G 1(1)=2 V(2) V^{2}(1), \\
& G 1(2)=3 V(3) V^{2}(1)+4 V^{2}(2), \\
& G 1(3)=4 V(4) V^{2}(1)+12 V(2) V(3), \\
& G 1(4)=5 V(5) V^{2}(1)+16 V(2) V(4)+9 V^{2}(3) \\
& G 1(5)=6 V(6) V^{2}(1)+24 V(3) V(4)+20 V(2) V(5), \\
& G 1(6)=7 V(7) V^{2}(1)+16 V^{2}(4)+30 V(3) V(5)+24 V(2) V(6), \\
& G 2(0)=U^{2}(0) \\
& G 2(1)=2 U(1) U(0), \\
& G 2(2)=2 U(2) U(0)+U^{2}(1), \\
& G 2(3)=2 U(3) U(0)+2 U(1) U(2), \\
& G 2(4)=2 U(4) U(0)+2 U(1) U(3)+U^{2}(2) \\
& G 2(5)=2 U(5) U(0)+2 U(2) U(3)+2 U(1) U(4),
\end{aligned}
$$




$$
\begin{aligned}
& G 2(6)=2 U(6) U(0)+U^{2}(3)+2 U(2) U(4)+2 U(1) U(5), \\
& G 3(0)=V^{2}(0), \quad G 3(1)=2 V(1) V(0), \quad G 3(2)=2 V(2) V(0)+V^{2}(1), \\
& G 3(3)=2 V(3) V(0)+2 V(1) V(2), \\
& G 3(4)=2 V(4) V(0)+2 V(1) V(3)+V^{2}(2), \quad G 3(5)=2 V(5) V(0)+2 V(2) V(3)+2 V(1) V(4) \text { and } \\
& G 3(6)=2 V(6) V(0)+V^{2}(3)+2 V(2) V(4)+2 V(1) V(5) .
\end{aligned}
$$

Using the recurrence relations in system (44), the transformed initial conditions in (45), and the relations in equations (46)-(48), one can easily evaluate $U(k)$ and $V(k)$. Hence, utilizing the inverse rule in (2), the series solution of the system (42) up to $O\left(x^{10}\right)$ is given by

$$
\begin{aligned}
& u(x)=1+2 x+\frac{x^{2}}{2}+\frac{x^{3}}{6}+\frac{x^{4}}{24}+\frac{x^{5}}{120}+\frac{x^{6}}{720}+\frac{x^{7}}{5040}+\frac{x^{8}}{40320}+\frac{x^{9}}{362880}+O\left(x^{10}\right) \text { and } \\
& v(x)=-1-\frac{x^{2}}{2}-\frac{x^{3}}{6}-\frac{x^{4}}{24}-\frac{x^{5}}{120}-\frac{x^{6}}{720}-\frac{x^{7}}{5040}-\frac{x^{8}}{40320}-\frac{x^{9}}{362880}-O\left(x^{10}\right) .
\end{aligned}
$$

For sufficiently large number of terms, the closed form of the solution is $u(x)=x+e^{x}$ and $v(x)=x-e^{x}$ which is the exact solution. Table 5 shows the absolute relative error (Abs. rel. err.) obtained for three various numbers of terms and at some test points.

Table 5: Numerical comparison of results in Example 6.

\begin{tabular}{|l|l|l|l|}
\hline$x$ & Abs. rel. err., (5 Terms) & Abs. rel. err.,(10 Terms) & Abs. rel. err., (15 Terms) \\
\hline 0.2 & $6.43685 \mathrm{E}-08$ & 0 & 0 \\
\hline 0.4 & $3.18791 \mathrm{E}-06$ & $5.74529 \mathrm{E}-13$ & 0 \\
\hline 0.6 & $2.92308 \mathrm{E}-05$ & $3.94906 \mathrm{E}-11$ & 0 \\
\hline 0.8 & 1.35599 E-04 & $7.61776 \mathrm{E}-10$ & 0 \\
\hline
\end{tabular}

\section{Conclusion}

In this work, we presented a new approach for applying the modified differential transform method for solving nonlinear integro-differential equations. The differential transform of the nonlinear term is replaced in the recurrence relation by its Adomian polynomial of index $k$. Hence, the dependent variable components are replaced by their corresponding differential transforms of the same index. The considered test examples include Volterra, Fredholm and coupled system of integro-differential equations with different types of nonlinearity. From these examples, the presented technique generated numerical results and is effective in solving nonlinear integro-differential equations.

\section{References}

[1] P. K. Kythe, P. Puri, Computational Methods for Linear Integral Equations, University of New Orleans, New Orleans 1992.

[2] A. M. Wazwaz, A comparison study between the modified decomposition method and traditional method, Appl. Math. Comput.181 (2006) 1703-1712. 
[3] M. T. Rashed, Numerical solution of functional differential, integral and integro-differential equations, Appl. Numer. Math. 156 (2004) 485-492.

[4] M. Razzaghi, S. Yousefi, Legendre wavelets method for nonlinear Volterra-Fredholm integral equations, Math. Comput. Simul. 70 (2005)1-8.

[5] K. Maleknejed, F. Mirzaee, Numerical solution of integro-differential equations by using rationalized Haar functions method, Kybernetes Int. J. Syst. Math. 35 (2006) 1735-1744.

[6] M. H. Reihani, Z. Abadi, Rationalized Haar functions method for solving Fredholm and Volterra integral equations, J. Comput. Appl. Math. 200 (2007) 12-20.

[7] P. Darania, E. Abadian, A. V. Oskoi, Linearization method for solving nonlinear integral equations, Math. Probl. Eng. (2006) 1-10 (doi: 10.1155/MPE/2006/73714)

[8] J. Zhao, R. M. Corless, Compact finite difference method for integro-differential equations, Appl. Math. Comput. 177 (2006) 271-288.

[9] S. Abbasbandy, A. Taati, Numerical solution of the system of nonlinear Volterra integro-differential equations with nonlinear differential part by the operational Tau method and error estimation, J. Comput. Appl. Math. 231 (2009) 106-113

[10] G. Ebadi, M. Y. Rahimi-Ardabili, S. Shahmorad, Numerical solution of the nonlinear Volterra integro-differential equations by the Tau method, Appl. Math. Comput. 188 (2007) 1580-1586.

[11] K. Maleknejad, B. Basirat, E. Hashemizadeh, Hybrid Legendre polynomials and Block-pulse functions approach for nonlinear VolterraFredholm integro-differential equations, Comput. Math. Appl. 61 (2011) 2821-2828.

[12] A. M Wazwaz, The combined Laplace transform-Adomian decomposition method for handling nonlinear Volterra integro-differential equations, Appl. Math. Comput. 216 (2010) 1304-1309.

[13] M. A. F. Araghi Sh. S. Behzadi, Solving nonlinear Volterra-Fredholm integro-differential equations using the modified Adomian decomposition method, Comput. Meth. Appl. Math. 9 (2009) 321-331.

[14] P. Darania, K. Ivaz, Numerical solution of nonlinear Volterra-Fredholm integro-differential equations, Appl. Math. Comput. 56 (2008) 2197-2209.

[15] K. Maleknejad, Y. Mohmoudi, Taylor Polynomial solution of high-order nonlinear Volterra-Fredholm integro-differential equations, Appl. Math. Comput. 145 (2003) 641-653.

[16] S. Yalcinbas, Taylor polynomial solution of nonlinear Volterra-Fredholm integral equations, Appl. Math. Comput. 127 (2002) 195-206.

[17] A. Borhanifar, R. Abazari, Differential transform method for a class of nonlinear integro-differential equations with derivative type kernel, Canad. J. Comput. Math., Natural Sciences, Eng. and Mid. 3 (2012) 1-6.

[18] Arikoglu, I. Ozkol, Solution of boundary value problems for integro-differential equations by using differential transform method, Appl. Math. Comput. 168 (2005) 1145-1158.

[19] Arikoglu, I. Ozkol, Solution of integral and integro-differential equation systems by using differential transform method, Comput. Math. Appl. 65 (2008) 2411-2417.

[20] Z. M. Odibat, Differential transform method for solving Volterra integral equation with separable kernels, Math. Comput. Model. 48 (2008) 1144-1149.

[21] J. Biazar, M. Eslami, Differential transform method for systems of Volterra integral equations of the second kind and comparison with homotopy perturbation method, Int. J. Phys. Sci. 6 (2011) 1207-1212.

[22] J. K. Zhou, Differential Transformation and Its Applications for Electrical Circuits, Huazhong University Press, Wuhan, China 1986.

[23] S. H. Chang, I. L. Chang, A new algorithm for calculating one-dimensional differential transform of nonlinear functions, Appl. Math. Comput. 195 (2008) 977-808.

[24] A. Elsaid, Fractional differential transform method combined with Adomian polynomials, Appl. Math. Comput. 218 (2012) 6899-6911.

[25] J. S. Duan, Convenient analytic recurrence algorithm for Adomian polynomials, Appl. Math. Comput. 218 (2011) 6337-6348.

[26] J. S. Duan, Recurrence triangle for Adomian polynomials, Appl. Math. Comput. 216 (2010) 1235-1241.

[27] J. S. Duan, An efficient algorithm for the multivivariable Adomian polynomials, Appl. Math. Comput. 217 (2010) 2456-2467.

[28] G. Adomian, Solving frontier problems of physics:The decomposition method, Kluwer Academic Publishers, MA, 1994.

[29] J. Biazar, H. Ghazvini, M. Eslami, He's homotopy perturbation method for systems of integro-differential equations, Chaos, Solitons and Fractals, 39 (2009) 1253-1258. 